\title{
Synchronized Communication in Low Power WBAN
}

\author{
Nathirulla Sheriff \\ Department of Electrical Engineering \\ Jonkoping University \\ Jonkoping, Sweden
}

\author{
Naveed Nawaz \\ Department of Computer Engineering \\ American University of Sharjah \\ Sharjah, UAE
}

\begin{abstract}
Wireless Body Area Network (WBAN) requires efficient and innovative solutions for synchronized communication and to meet the energy consumption requirements for independent sensor nodes. In any wireless communication, the low message rate gives low power consumption but there is always a trade-off between power consumption and dynamic response. This paper presents the time synchronization methods and to develop a prototype to receive the time synchronized data in WBAN server from ANT wireless sensor nodes in SIGS system. The message rate used in SIGS is very low with $8 \mathrm{~Hz}$, to consume less power. Hence it is essential to synchronize the system at low message rate. Whenever a data is received at WBAN / Linux server through ANT protocol, the received data is difficult to handle because of unknown sampling time. To overcome this problem and to receive time synchronized data, the new methods and prototype was designed. The time synchronization methods implemented provided good time synchronization for ANT low power wireless sensor network.
\end{abstract}

\section{Keywords}

Wireless Body Area Network, Time Synchronization, Health care, Wireless Sensor Networks

\section{INTRODUCTION}

The use of wireless technology in the field of health care plays an important role and could be a possible solution to solve the wired connection problem. The use of a wireless interface for health care enables an easier application and is more cost efficient. The wireless technology could help the patient to experience a greater physical mobility and they are no longer compelled to stay in a hospital. This present trend could replace the bottlenecks in the past and could provide a greater enhancement for personal health care with low costs of the health care system.

In order to utilize the benefits of wireless technologies in telemedicine and for mobile health care services in an efficient way, a new type of wireless network emerges: a wireless on-body network or a wireless body area network (WBAN) [1]. Short range wireless data communication networks that are used for sport and health care are sometimes called wireless body area networks (WBANs) and they are located more or less on a person. In WBAN, various sensors are attached on clothing or on the body or even implanted under the skin. The wireless nature of the network and the usage of wide variety of sensors in WBAN provide an environment to develop many new, practical and innovative applications to improve the health care.
Sole Integrated Gait Sensor (SIGS) is a research project to develop a foot pressure activated feedback system for enhancing static and dynamic balance in elderly subjects who have suffered from a stroke [7]. In SIGS, the sampled data need to be synchronized because of low message rate. The low message rate gives low power consumption but there is a trade-off between power consumption and dynamic response. This paper presents a method to find and develop a prototype to receive the time synchronized data in WBAN server from ANT wireless sensor nodes in SIGS system.

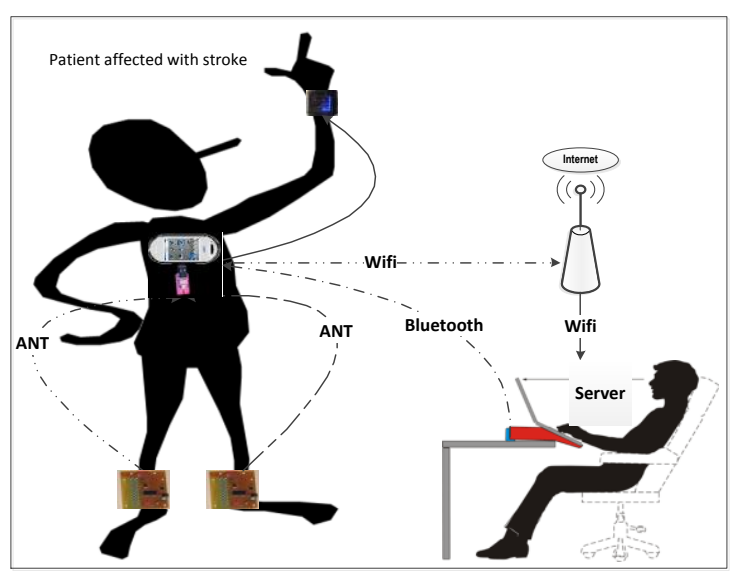

Figure 1. Architecture of WBAN System

The rest of the paper is organized as follows. Section II shows a brief introduction to ANT Protocol and SIGS system. In Section III the design and implementation of the time synchronization protocol were discussed. The performance analysis and the implementation results are presented in Section IV and finally the conclusion under Section V.

\section{ANT PROTOCOL AND SYSTEM ARCHITECTURE}

\subsection{ANT Protocol}

ANT is a practical and a proprietary wireless sensor network protocol [4]. Its protocol stack enables the semiconductor radios to operate in $2.4 \mathrm{GHz}$ ISM band. The protocol is designed and marketed by Dynastream Innovations Inc., Canada. Its design is suited for any kind of low data rate sensor network topologies in practical wireless sensor networks (WSN), Wireless Body Area Networks (WBAN) and Personal Area Networks (PAN). All ANT powered network nodes can operate for years as compared to months for other technologies because of its energy efficient protocol. 
In the OSI layer model of ANT shown in the figure 2, the protocol provides efficient handling of the Data-link, Network and Transport layer along with the physical layer provided by the Nordic $2.4 \mathrm{GHz}$ radio [4]. The top level Session, Presentation and Application layers are user-defined. The interface design between the ANT and Host application are made simpler for quick and easy implementation of ANT with new devices and applications.

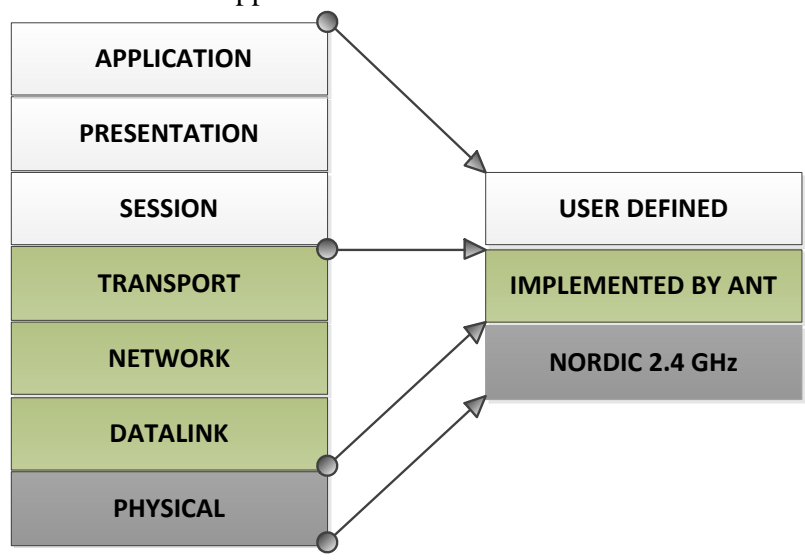

Figure 2: OSI layer model of ANT Protocol

The type of communication between nodes in ANT channel is determined by ANT data types such as Broadcast, Acknowledgement and Burst transfers. The data messages between nodes are transferred in Forward (Master to Slave) or Reverse (Slave to Master) direction. Once the channel is opened for communication, a master device will transmit a message on each channel in their allocated time slot. Broadcast data is the most basic and system default data type. On every timeslot, the broadcast data is always sent from Master node to Slave and vice versa only when there is a request from the slave's host MCU.

Broadcast data is never acknowledged without any awareness of data loss. It consumes very less power and least amount of RF bandwidth because of one way transmission. For a practical wireless network, the batteries powering nodes need to last for months (or even years) to minimize maintenance. For this purpose, Low power consumption is very essential.

\subsection{SIGS}

Sole Integrated Gait Sensor Analysis (SIGS) is a research project to develop a foot pressure activated feedback system for enhancing static and dynamic balance in elderly subjects who have suffered from a stroke. The research was started, to design and built a system which has the ability to measure and provide immediate feedback to patients regarding the distribution of weight through their feet. It has enormous potential for the rehabilitation industry and prevention injuries.

In this project, wireless pressure sensors are placed like soles in the shoes of persons with different kinds of deceases. The sensors can measure the pressure of the foot relative the shoe i.e. the load of the two legs is measured. This information can be useful e.g. to not over or under load a leg after joint replacement or as a bio feedback system to help e.g. post stroke patients to avoid falling.

\subsection{Architecture}

The system was built in a star topology WBAN fashion which includes a central node and two leaf nodes as shown in Figure 1.The central node is a personal server which is NeoFreeRunner Smartphone along with an ANT USB stick transceiver unit. The central node could be attached to the user's belt or it can be hanged on the user's neck. On the other side, the leaf nodes or SIGS consist of a foot pressure sensor and an ANT transceiver unit. The leaf node will continuously transmit data to the central node whenever they are active ie., triggered by the movement sensors.

In this research, the Neo FreeRunner Smartphone [3] is used as a personal server whose function involves the data analysis and to generate the biofeedback signals like audio warning signal. It also acts as a bridge between the WBAN and the home server as shown in the figure 1. The receiver ANT (Nordic USB ANT stick) is attached with the Smartphone through USB port. The SIGS system acts as a leaf node which consists of a wireless transceiver unit and the foot pressure sensor from Tekscan [5]. In this system, the Tekscan sensor is used together with the wireless sensor node to transmit the sensor data to the personal server.

\section{DESIGN AND IMPLEMENTATION}

\subsection{System Design}

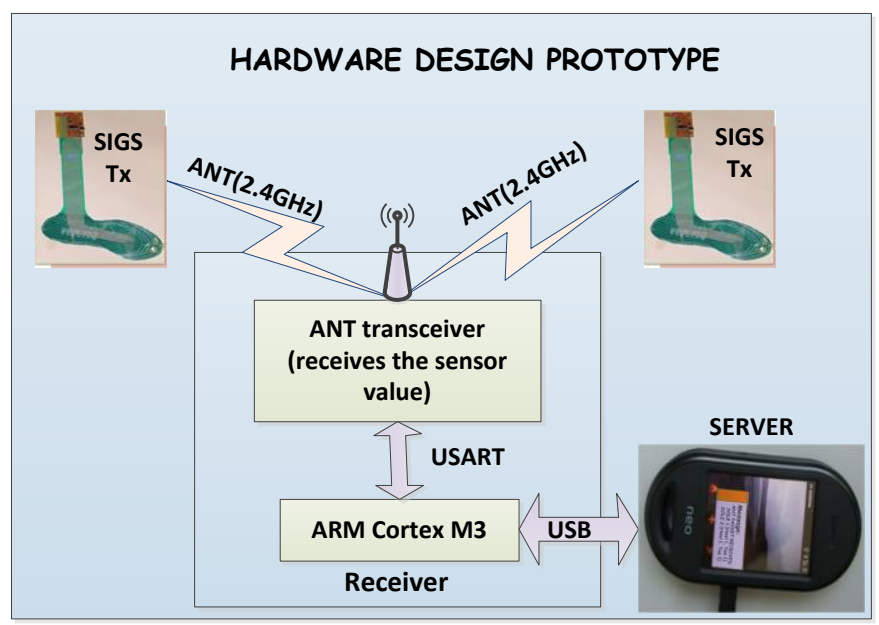

Figure 3: Hardware design for WBAN

The SIGS system acts as a leaf node which consists of a wireless transceiver unit and the foot pressure sensor from Tekscan [5]. In this system, the Tekscan sensor is used together with the wireless sensor node to transmit the sensor data to the personal server. The system is used in our implementation phase for the transmission of message packet from the sole pressure sensors to the WBAN server. It consists of a control board with Nordic Semiconductor nRF24L01 which uses ANT proprietary protocol, Atmel ATmega88 [10] microcontroller and small coin cell battery.

This is a small breakout board with circuit for the Nordic nRF24AP1 transceiver [2]. The transceiver IC is capable of talking with other wireless products which is built on ANT protocol. The wireless transceiver unit as shown in the Figure 3 receives the message packet from SIGS (Sole Integrated Gait Sensor) through ANT communication protocol and transmits the received message packet serially through USART to the ARM Cortex M3. 
The environment in which WBAN server implemented is Linux PC for testing. In real-time, Neo-FreeRunner Smartphone was used which runs on Linux OS. The server was designed and programmed such that it could communicate with the designed prototype through USB.

\subsection{Time Synchronization}

For synchronization between two nodes, the ANT protocol uses TDMA techniques for its communication channels. TDMA techniques combined with the ANT multiple access channel technology plays a major role for the users to use two to thousands of nodes to be connected to an ANT network [4]. In communication between two nodes, the messages are transmitted in forward direction at designated channel period. Once the channel is opened, the master will send the message in its allocated time slot and wait for the next allocated time slot to sent.

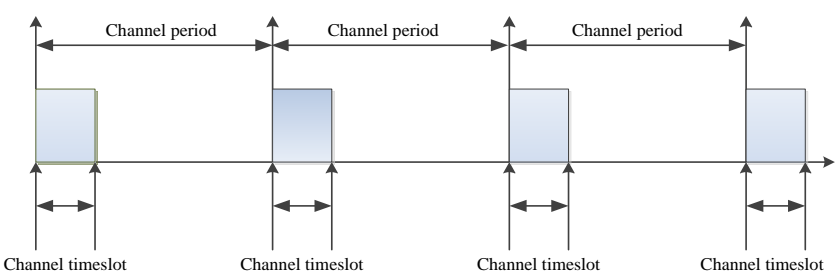

Figure 4: TDMA technique in ANT protocol

If two nodes try to transmit the messages to receiver, both the nodes sent the messages at their allocated time slots. As the protocol is proprietary and the scheduling of time slots is adaptive isochronous co-existence i.e. the scheduling is dynamic. ANT uses an adaptive isochronous scheme which implies that any free time slot can be used. In the data payload two bytes are reserved for source time stamping and the remaining four bytes are used for sole load data.

\subsubsection{Sequence number}

To take care of lost data in SIGS system the packets are given sequence numbers when they are sent from the sole sensors. But there is no improvement of synchronization time skew with this method.

\subsubsection{Time Stamp Protocol}

The sensor data from the two soles are sampled before it is transmitted. The ANT transceiver attached to the Host sends the message packet with 11 bytes via radio channel and sends back the acknowledgement before the host goes into sleep mode at $\mathrm{T} 2$. The sensor data are sampled after the fixed delay $\mathrm{T} 2$ as shown in the figure. The transmitted packet will be received at the receiver host through ANT transceiver. As soon as the packet is received at the ANT transceiver at the receiver host, the time stamping shall be done on the two bytes allocated on the received packet and its detailed operation is explained in the previous research work [8].

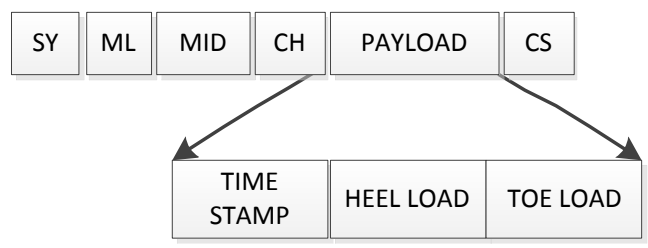

\begin{tabular}{|c|c|c|}
\hline Field Name & Bytes & Description \\
\hline SY & 1 & Sync, 0xa4 Fixed value \\
\hline ML & 1 & Length ,0x09 \\
\hline MID & 1 & Data type ID, 0x4e \\
\hline CH & 1 & Channel number \\
\hline Time stamp & 2 & ms(or sequence \#) \\
\hline Toe load & 2 & Raw sensor data \\
\hline Heal load & 2 & Raw sensor data \\
\hline CS & 1 & XOR of all previous data \\
\hline
\end{tabular}

Figure 5: Message structure of the data packet

Hence the transceiver could transmit before the start of the next channel period and thus the protocol could produce a better synchronization. Through experiments, it is noted that once the time slot is allocated for the channel at the start of the synchronization, it utilizes approximately the same allocated time slot at the time of re-synchronization after every packet loss. With time stamping it is possible to estimate the actual sampling point.

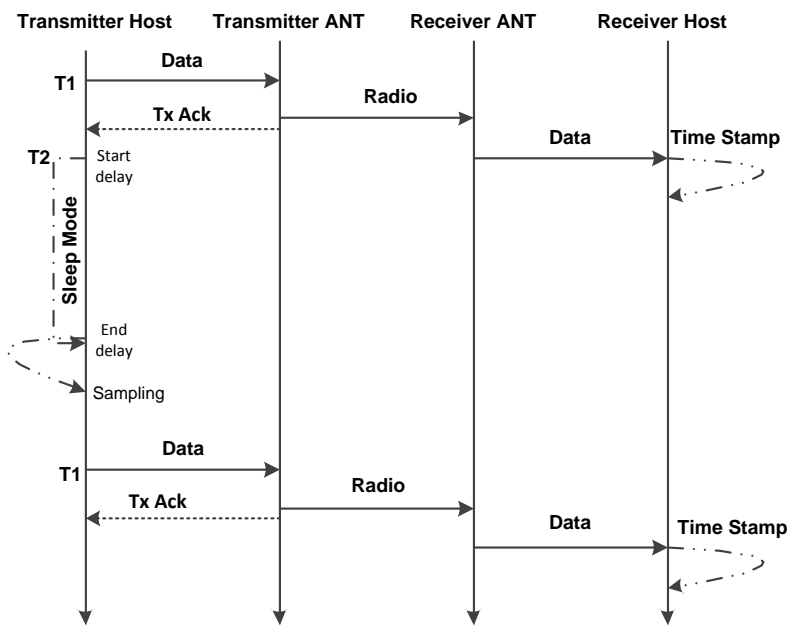

Figure 6: MSC for Time Stamp Protocol

\subsubsection{Global Synchronization}

In SIGS system, the implementation of global clock [6] synchronization could provide a possible solution for efficient synchronization. By time-stamping at the receiver the samplings are synchronized with the corresponding timeslot in the ANT-communication but its scheduling of time slots is adaptive isochronous co-existence i.e. the scheduling is dynamic, gives a varying delay between the two sensors. In order to synchronize the samplings of the two sole sensors without delays a global sample command is needed. 


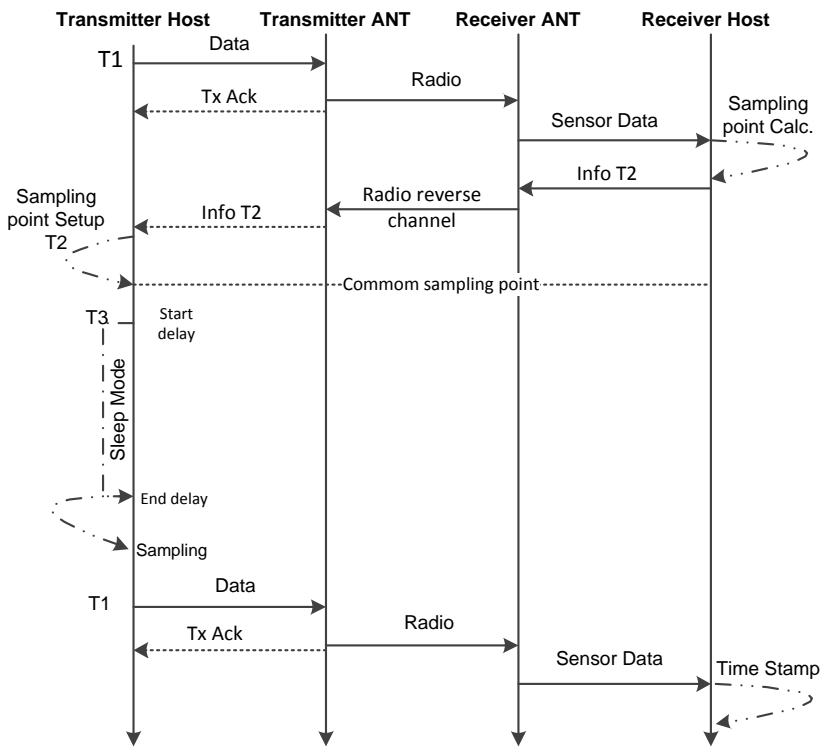

Figure 7: MSC for Global Synchronization

With no external disturbances in the SIGS system, the used time slots will not be disturbed until there is any clash between the two channels. The clock oscillators will not work as it needs to be in the SIGS host. So there are possibilities of interference in the time slots of the two channels. Therefore the refresh rate of the global clock in the communication cycle will be after every resynchronization interval. In our system [9], the maximum drift of the clock is approx. $50 \mathrm{ppm}$ as provided by the manufacturer. The maximum clock skew is about $1 \mathrm{~ms}$. Thus the clocks should resynchronize at the latest every,

$$
R_{\text {int }}=\left(1 * 10^{-3}\right) /\left(2 * 50 * 10^{-6}\right)=10 \text { sec approx }
$$

The clocks need to resynchronize after every 10 seconds and hence a global sample command shall be provided after every 10 seconds. For the implementation of Global synchronization, Broadcast data combined with reverse channel shall be used .It will increase the power consumption but relatively very little.If for example the reverse channel is used once per second then the increase in power consumption is less than $50 \mu \mathrm{A}$.

The Personal Server receives and time-stamps the data messages from the two sole sensors (Fig. 5) and from these time-stamps calculates a sampling point that has a minimum distance to the two time-slots used. The sampling point relative the time-slot is the delay T2. The Reverse Channel is used to send T2 (T2left and T2right) to the two sensors. T2 is the SIGS Host micro-controller sleep time before sampling the sensor and $\mathrm{T} 3$ after sampling the sensor.

\section{PERFORMANCE ANALYSIS}

\subsection{Functionality test}

The SIGS system has been tested under different conditions. The main focus has been on synchronization of data streams from two sole sensor nodes, but the system functionality in general is also tested. The ANT protocol is robust and can coexist with WiFi/BlueTooth/GSM/3G. The Message Error Rate is low enough for the application.

\subsection{Performance test}

\subsubsection{Sequence Numbers and Time-stamping at Receiver}

Figure 8 shows a short sequence of a varying load [8]. As the delay between the signals from left and right foot can be calculated it is possible to estimate new signals with zero delay. In order to estimate the new signal values with zero delay, linear interpolation shall be used. The signal from the two sole sensors shall be considered, with one as the reference signal and the other signal shall interpolate to match the sampling points. Consider two sampled signals from the samples of two soles as Left sole [X (n)] and Right sole [Y (n)]. The time synchronization error between the sampled signals shall be considered as $\Delta \mathrm{t}$. The time difference between the two samples in the SIGS system is T. The new corrected signal shall be calculated from the below equation,

$$
C(n)=X(n)+\frac{\Delta t}{T} \times \frac{\{X(n+1)-X(n-1)\}}{2}
$$

From the experiments, the time synchronization error between the two samples soles is $55 \mathrm{~ms}$; such that the right sole is sampled $55 \mathrm{~ms}$ before the left sole. The sampled signal from left sole is considered as the reference signal and the sampled signal from the right sole shall interpolate to match sampling points. In figure 8 , the sampled signal from the right sole is moved $55 \mathrm{~ms}$; such that both the soles are sampled at the same time. The new corrected signal is estimated using the above mentioned equation. The new corrected sampling points for all the values in the right sensor are estimated and plotted in the graph as shown in the figure 8.

The root mean square errors (RMSE) for corrected and uncorrected right sensor are estimated using the equation as shown below.

$$
R M S E=\sqrt{\frac{\sum f\{[X(n)]-[Y(n)]\}^{2}}{i}}
$$

Where,

$\mathrm{X}(\mathrm{n}) \quad$ - Reference sampled signal from left sensor

Y (n) - Corrected / Uncorrected sampled signal from right sensor

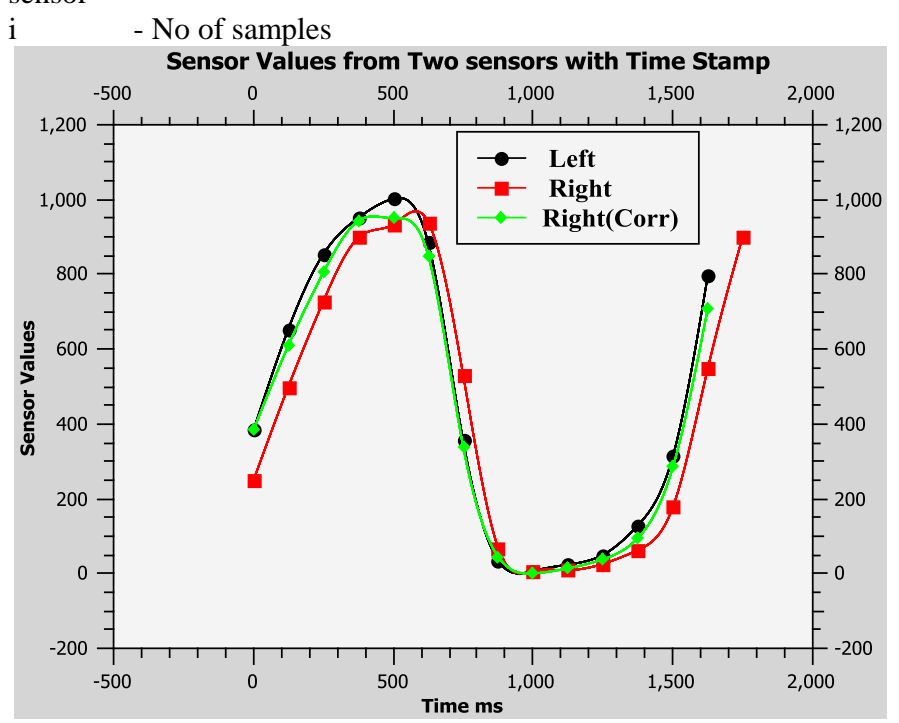

Figure 8:Time Stamping at Receiver 
For uncorrected right sensor with sequence number, the RMSE $=10.6 \%$, and for the corrected right sensor with time stamp protocol, the RMSE $=2.9 \%$.

\subsubsection{Global Synchronization}

The receiver is programmed in such a way that it could calculate and send the T2left and T2right on ANT reverse channel to the two sole nodes to get concurrent sampling of the sensors.

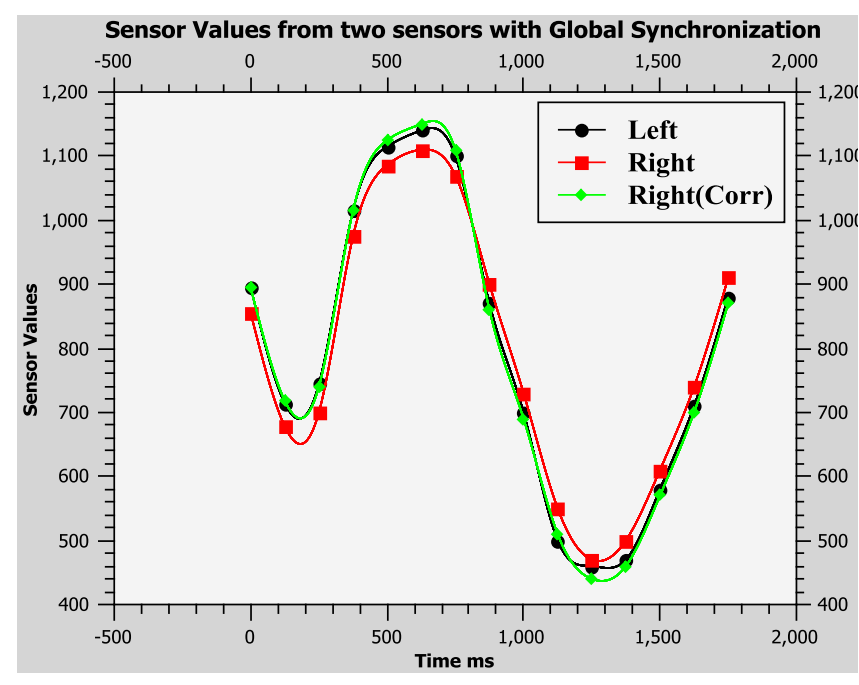

Figure 9: Global Synchronization

The right sensor values Right (corrected) are only compensated for a small difference in gain. The new corrected sampling points for all the values in the right sensor are only estimated for a small difference in gain and plotted in the graph as shown in the Figure 9.Figure 9 shows the good correlation and the RMSE is $2.3 \%$.

\section{CONCLUSION}

In this paper, Time synchronization for ANT based Wireless Body Area Network (WBAN) has been presented. As the ANT protocol is a proprietary protocol, the scheduling of time slots is adaptive isochronous co-existence i.e. the scheduling is dynamic and unpredictable. In this paper, the designed synchronization method was implemented to know about the time synchronization in ANT protocol and to provide a time synchronized data to the ANT receiver. The implemented protocol provides us the packet data with time stamp through which we could know when the data is measured. The TDMA access method gives little time jitter for the data sampling but it must be complemented with time stamping to take care of lost data frames and time-synchronization skew. Global synchronization method gives the best matching between the sensors but time-stamping at receiver and re-sampling of one of the sensors also gives good matching. Moreover the data with time stamp helped the receiver to store the data in its buffer and made easier for future reference. The implemented time synchronization method in SIGS is intended to be a research project to develop a foot pressure activated feedback system for enhancing static and dynamic balance in elderly subjects who have suffered from a stroke.

\section{REFERENCES}

[1] Benoit Latre, Bart Braem, Ingrid Moerman, Chris Blondia,Piet Demeester. A Survey on Wireless Body Area Networks. Available online at http://www.pats.ua.ac.be/content/publications/2010/bbra em10wbansurvey.pdf

[2] nRF24AP1 Nordic Semiconductor, Product Specification. Available online at http://www.sparkfun.com/datasheets/Wireless/Nordic/nR F24AP1.pdf

[3] NeoFreeRunner Smartphone, Features and Specifications. Available online at http://wiki.openmoko.org/wiki/Neo_FreeRunner\#Specifi cations

[4] ANT Message Protocol and Usage. Available online at http://www.thisisant.com

[5] F-SCAN Foot Pressure Sensor, Data Sheet. Available online at http://www.tekscan.com

[6] Kopetz, Hermann. Real-Time Systems: Design Principles for Distributed Embedded Applications. Pp 45-68.

[7] Whittle, Michael W. Gait Analysis: An Introduction. 4th Edition. Edinburgh: Elsevier Butterworth Heinemann, 2007.

[8] Nathirulla sheriff, Naveed Nawaz .Time Stamp Performance in Low Power Bio-Feedback Network. Available online at http://www.europeanjournalofscientificresearch.com/ISS UES/EJSR_65_3_09.pdf.

[9] STM32-32 bit-ARM CORTEX microcontroller Available online at ,http://www.st.com/internet/mcu/class/1734.jsp

[10] Atmel ATmega88 Datasheet, Available online at http://www.atmel.com/dyn/resources/prod_documents/do c2545.pdf 